International Journal of Tropical Medicine 5 (1): 6-9, 2010

ISSN: 1816-3319

(C) Medwell Journals, 2010

\title{
Plasma Transferrin and Caeruloplasmin Levels in Nigerians with Diabetes Mellitus
}

\author{
${ }^{1}$ V.O. Mabayoje, ${ }^{2}$ E.O. Akanni, ${ }^{3}$ G.O. Arinola and ${ }^{4}$ R.O. Hassan \\ ${ }^{1}$ Department of Haematology and Blood Transfusion, \\ ${ }^{2}$ Department of Biomedical Sciences, College of Health Sciences, \\ Ladoke Akintola University of Technology, P.M.B. 4400 Osogbo, Osun State, Nigeria \\ ${ }^{3}$ Department of Chemical Pathology, University of Ibadan, Ibadan Oyo State, Nigeria \\ ${ }^{4}$ Department of Haematology, Teaching Hospital, \\ Ladoke Akintola University of Technology, P.M.B. 5000 Osogbo, Osun State, Nigeria
}

\begin{abstract}
Transferrin and ceruloplasmin levels in Nigerians diagnosed with diabetes mellitus were studied. Eighty subjects consisting of 40 diabetes mellitus patients and 40 apparently healthy individuals who served as controls were involved. Transferrin and ceruloplasmin levels were estimated using single radial immuno diffusion method. Results showed that Ceruloplasmin levels were significantly higher $(p<0.05)$ in diabetic patients than in control subjects. Transferrin levels were on the other hand significantly lower $(p<0.05)$ in diabetic patients when compared to the controls. The study reveals that both Ceruloplasmin and transferrin levels in Nigerian diabetes patients may be useful markers in monitoring and controlling diabetes mellitus.
\end{abstract}

Key words: Transferrin, ceruloplasmin, diabetes mellitus, immuno-difussion, Nigeria

\section{INTRODUCTION}

Diabetes mellitus is a group of metabolic disorders characterized by high blood sugar (glucose) levels that result from defects in insulin secretion or action or both. Diabetes mellitus was first identified as a disease associated with sweet urine and excessive muscle loss in the ancient world. Elevated blood glucose levels (hyperglycaemia) leads to spillage of glucose into the urine, hence the term sweet urine (Ponder et al., 2000). It is a major non-communicable disease with a higher incidence in the developed countries (WHO, 1985). The characteristics symptoms are excessive urine production, excessive thirst, pruritus and often unexplained loss of weight (Cheesbrough, 2000).

The World Health Organization (WHO, 1999) recognizes three main forms of diabetes mellitus: type I, type II and gestational diabetes which have different causes and population distribution. Type 1 diabetes is usually due to auto-immune destruction of the pancreatic beta cells leading to absolute insulin deficiency (Ponder et al., 2000).

Type II diabetes is the most common type found in about $90 \%$ of those with diabetes mellitus. Obesity is common in patients with type II diabetes (ADA, 2000). Gestational diabetes mellitus is a carbohydrate intolerance of varying degrees with onset or first recognition during pregnancy (Metzger and Coustan, 1998). Diabetic women who become pregnant are not included.

The aetiology of diabetes is usually due to production of insufficient insulin, defective insulin or inability of cells to utilize glucose effectively, leading to diabetes. The latter is the primary problem in type II diabetes. The absolute lack of insulin due to a destructive process affecting beta cell in the pancrease is the main disorder of type 1 diabetes (Ponder et al., 2000).

Ceruloplasmin (CP) is an " -2 globulin that contains approximately $95 \%$ of total copper found in serum. Each molecule of CP contains 6-8 copper atoms, most are tightly bound. CP is synthesized primarily by the hepatic paranchymal cells, with small amounts synthesized by macrophages and lymphocytes. Its primary physiological role involves plasma redox reaction. Transferrin is the principal plasma transport protein for $\mathrm{Fe}^{3+}$ (iron). It is a single polypeptide chain with carbohydrate moieties in the c-terminal and two homologous domains each containing an iron binding $\left(\mathrm{Fe}^{3+}\right)$ site (Van Eden and Young, 1995). It is synthesized almost exclusively in the liver, with lesser amount in the choroids plexus of the brain. An increase in serum CP level has been reported in type II D.M. CP permits the incorporation of iron into transferrin. From previous studies, 50 patients with type II DM and 21 clinically healthy subjects were evaluated. Patients were divided into two groups. Group 1 included

Corresponding Author: V.O. Mabayoje, Department of Haematology and Blood Transfusion, College of Health Sciences, Ladoke Akintola University of Technology, P.M.B. 4400 Osogbo, Osun State, Nigeria 
29 patients without diabetic complications. Group 2 included 21 patients with diabetic complications. Serum $\mathrm{CP}$ and transferrin levels were studied. Serum CP in diabetic patients was significantly higher than those of controls (Ramaza and Ebukekir, 2004). Transferrin levels were significantly lower in diabetic patients than those of controls. CP levels in group 2 were significantly higher than those of group 1 (Ramaza and Ebukekir, 2004).

This study aimed at determining the relationship between diabetes mellitus and plasma transferrin and ceruloplasmin levels in Nigerian patients.

\section{MATERIALS AND METHODS}

Study site: This study was conducted at Osogbo (Osun State Capital), South West Nigeria (Longitude $4^{\circ} 57^{\prime} \mathrm{E}$ and Latitude $7^{\circ} 77^{\prime} \mathrm{N}$ ). It is the commercial city of the state. Osogbo is being served by two major government hospitals (Ladoke Akintola University of technology (LAUTECH) Teaching Hospital (LTH) and the State Hospital, (Asubiaro) and other privately owned Hospitals. The city is $342 \mathrm{~km}$ South of Abuja (the Federal Capital Territory (FCT) of Nigeria) and $194 \mathrm{~km}$ North of Lagos.

Subjects' selection: Non smoking subjects diagnosed of diabetes mellitus at the metabolic units of chemical pathology departments of the above health facilities that gave their consent among those that visited the centers between April and July, 2009 were involved in the study. Immunosuppressed patients with those that refused to give consent were excluded.

Ethical approval: Ethical clearance for the study was obtained from the ethical review committee of the Ladoke Akintola University of Technology (LAUTECH) and LAUTECH Teaching Hospital (LTH) Osogbo, Nigeria.

Samples collection: Five milliliters of venous blood was withdrawn from the ante-cubital vein of the fore-arm from each subject and was put into a bottle containing lithium heparin and spun at 3,000 rpm for 5 min to obtain plasma. The plasma was separated into new sterile bottles, stored and at $-20^{\circ} \mathrm{C}$ until analyzed.

Analytical method: Transferrin and ceruloplasmin levels were estimated using single radial Immuno-diffusion method of Fahey and McKelvey (1965) as modified by Salimonu et al. (1978) that are based on the antigen antibody precipitation reaction in agar gel.

Principle: When antigen and antibody diffuse towards one another in agar or when antibody is incorporated into the agar and antigen is allowed to diffuse into an antibody containing agar gel, a visible line of precipitation (zone of equivalence concentration) will form.
Materials, reagents and equipment: Anti-human transferrin Antibody (batch no: $\mathrm{N}$ anti-serum to human transferrin code no: OSAM from Dade Behring of Germany); Anti-human ceruloplasmin Antibody (batch no: $\mathrm{N}$ anti-serum to human ceruloplasmin code no: OUIE from Dade Behring of Germany).

Others include: noble agar, precision viewer, leveler, punch template, suction pump. Glass slide and phosphate buffered saline at $\mathrm{pH}$ 7.2. Phosphate Buffer Saline PBS (Sodium chloride $8.00 \mathrm{~g}$, Potassium chloride $0.30 \mathrm{~g}$, disodium hydrogen phosphate $1.15 \mathrm{~g}$ and Potassium dihydrogen phosphate $0.20 \mathrm{~g}$, all dissolved in $100 \mathrm{~mL}$ of distilled water.

Three percent noble agar was prepared in phosphate buffered saline PBS pH 7.2 containing $0.2 \%$ sodium-azide as preservative. One milliliter of anti-human transferrin and anti-human ceruloplasmin were mixed with $7 \mathrm{~mL}$ of PBS in a clean glass tube.

Eight milliliters of the prepared 3\% noble agar was measured into a long glass tube and thoroughly mixed with the diluted antiserum. The mixture was carefully poured onto a glass plate placed on a leveler, avoiding formation of air bubbles. The agar antiserum mixture was allowed to set and wells of $3 \mathrm{~mm}$ in diameter were made in the agar with a circular metal punch. The punched agar was carefully removed from the plate with the smooth edge of Pasteur pipette attached to a vacuum pump, taking care not to damage the sides of the wells.

Several dilutions $(25,50,100$ and $200 \%)$ of the standard serum were prepared in PBS. Using a $5 \mu \mathrm{L}$ micro-dispenser, both the sera and standard were applied to the punched wells. The plate for transferrin and ceruloplasmin were put in a humid chamber and incubated for $18 \mathrm{~h}$ at $4-8^{\circ} \mathrm{C}$. The diameter of the precipitation ring was measured along two perpendicular diagonals to the nearest $0.1 \mathrm{~mm}$ using eye precision viewer. The standard curves for transferrin and ceruloplasmin were plotted on a semi log graph paper and the concentrations of the test and control samples were read off the standard curve (Salimonu et al., 1978).

Statistical analysis: Student t-test and ANOVA were used to analyze the differences between the results.

\section{RESULTS AND DISCUSSION}

The results of the ceruloplasmin and transferring levels obtained in the study are as shown in the Table 1-3 and Fig. 1. The age of the diabetic patients is not significantly different from those of controls $(p>0.05)$, 
Table 1: Physical and biochemical parameters in diabetes patients and

\begin{tabular}{llllll}
\multicolumn{2}{c}{ controls } & & & & \\
\hline Parameters & Sex & $\mathrm{N}$ & Mean \pm SD & t-value & p-value \\
\hline Age (years) & Case & 40 & $46.1 \pm 8.1$ & & \\
& Control & 40 & $46.0 \pm 7.8$ & 0.06 & NS \\
CLP (g LG) & Case & 40 & $0.59 \pm 0.24$ & & \\
& Control & 40 & $0.22 \pm 0.07$ & 8.22 & $<0.01$ \\
TRF (g LG) & Case & 40 & $3.31 \pm 1.22$ & & \\
& Control & 40 & $3.79 \pm 0.92$ & 2.00 & $<0.05$ \\
\hline
\end{tabular}

Table 2: The sex distribution of the estimated parameters

\begin{tabular}{llllll}
\hline Parameters & Sex & N & Mean \pm SD & t-value & p-value \\
\hline Age (years) & Male & 16 & $51.4 \pm 4.8$ & & \\
& Female & 24 & $42.6 \pm 7.8$ & 4.42 & $<0.05$ \\
CLP (g LG) & Male & 16 & $0.42 \pm 0.14$ & & \\
& Female & 24 & $0.70 \pm 0.24$ & 4.0 & NS \\
TRF (g LG) & Male & 16 & $3.74 \pm 1.38$ & & \\
& Female & 24 & $3.05 \pm 0.98$ & 1.725 & NS \\
\hline
\end{tabular}

Table 3: Comparison of male subjects and male controls with all parameters

\begin{tabular}{llllll}
\hline Parameters & Sex & N & Mean \pm SD & t-value & p-value \\
\hline Age (years) & Male $_{(S)}$ & 16 & $51.4 \pm 4.8$ & & \\
& Male $_{(\mathrm{CN})}$ & 20 & $45.0 \pm 5.6$ & 3.69 & $<0.05$ \\
CLP (g LG) & Male $_{(\mathrm{SJ})}$ & 16 & $0.42 \pm 0.17$ & & \\
& Male $_{(\mathrm{CN})}$ & 20 & $0.20 \pm 0.10$ & 4.58 & $<0.05$ \\
\multirow{2}{*}{ TRF (g LG) } & Male $_{(\mathrm{SJ})}$ & 16 & $3.74 \pm 1.38$ & & \\
& Male $_{(\mathrm{CN})}$ & 20 & $4.30 \pm 2.30$ & -0.9 & $\mathrm{NS}$ \\
\hline
\end{tabular}

Table 4: Comparison of female subjects and female controls with all parameters

\begin{tabular}{llllll}
\multicolumn{2}{c}{ parameters } & & & \\
\hline Parameters & Sex & N & Mean \pm SD & t-value & p-value \\
\hline Age (years) & Female $_{(\mathrm{SJ})}$ & 24 & $42.6 \pm 7.8$ & & \\
& Female $_{(\mathrm{CN})}$ & 20 & $46.9 \pm 9.4$ & -1.63 & NS \\
CLP (g LG) & Female $_{(\mathrm{SJ})}$ & 24 & $0.70 \pm 0.24$ & & \\
& Female $_{(\mathrm{CN})}$ & 20 & $0.20 \pm 0.03$ & 10.2 & $<0.05$ \\
\multirow{2}{*}{ TRF (g LG) } & Female $_{(\mathrm{SJ})}$ & 24 & $3.05 \pm 0.98$ & & \\
& Female $_{(\mathrm{CN})}$ & 20 & $3.70 \pm 1.09$ & -2.06 & NS \\
\hline
\end{tabular}

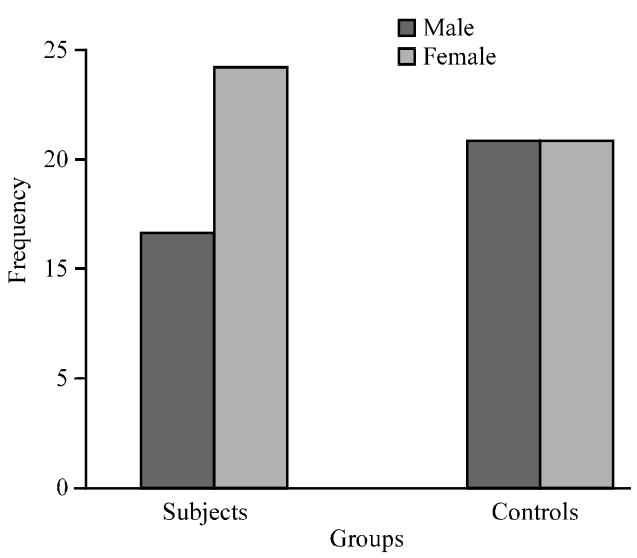

Fig. 1: Bar chart showing the gender distribution in the two groups

Table 1. The mean plasma concentrations of acute phase proteins, ceruloplasmin was significantly higher $(\mathrm{p}<0.01)$ in diabetic subjects in comparison to controls, while transferrin was significantly lower $(\mathrm{p}<0.05)$ in subjects when compared to controls (Table 1). When subjects were grouped into male and female, age in male test subjects was significantly different to that of the female subjects $(\mathrm{p}<0.05)$, Table 2. Table 2 shows that there was no significant difference $(p>0.05)$ in the mean plasma concentration of ceruloplasmin when male subjects were compared to female subjects. Also, when male subjects were compared to female subjects, the mean plasma concentration of transferrin showed no significant difference $(p>0.05)$.

However, comparison between male subjects and male controls was significant $(p<0.05)$, Table 3 . Table 3 shows that there was significant difference $(\mathrm{p}<0.05)$ in the mean plasma concentration of ceruloplasmin when male subjects were compared to male controls. However, when female subjects were compared to female controls there was no significant difference $(p>0.05)$ in transferrin. While comparison between female subjects and female controls was not significantly different (Table 4 ). Table 4 shows that there was significant difference $(\mathrm{p}<0.05)$ in the mean plasma concentration of ceruloplasmin when male subject were compared to male controls. However, when female subjects were compared to female controls there were no significant difference $(p>0.05)$ in transferrin.

Acute Phase Protein (APP) are classes of protein whose plasma concentration increases i.e., positive APP (Haptoglobin, ceruloplasmin, procalcitonin etc.) or decreases i.e., negative APP (Transferrin, albumin etc.) in response to inflammation (Killingsworth, 1979).

In this study, ceruloplasmin levels were significantly higher $(\mathrm{p}<0.05)$ in diabetes mellitus patients studied when compared to controls, while transferrin levels showed a significant decrease when compared to controls. This was also consistent with previous results by Ramaza and Ebukekir (2004). The rise in ceruloplasmin and fall in transferrin further confirms these proteins as positive and negative acute phase proteins.

Much research has not been done on these, but from this study, it was found that sex has no effect, while age did have an effect on the levels of ceruloplasmin and transferrin.

From previous research, increase ceruloplasmin and transferrin level in females was seen in pregnant women and those using oral contraceptives (estrogen) (Yonekawa et al., 1999). Studies carried out by Yoshida et al. (1995) and Yonekawa et al. (1999) showed that ceruloplasmin levels were decreased as a result of various primary (genetic) deficiencies. Inherited deficiencies of ceruloplasmin have been reported in several families. Clinical findings in homozygous individuals include diabetes mellitus, dementia, blepharospasm etc. No patients have shown excessive tissue copper, but all have had increase tissue iron stores and decrease serum iron. This argues strongly for the hypothesis that the primary role of ceruloplasmin is for 
maintaining normal iron transport and metabolism (Yoshida et al., 1995; Yonekawa et al., 1999). Serum ceruloplasmin levels reach a maximum at 2 or 3 years of age and then fall slowly until the adolescent years, when adult levels are reached. Levels are higher in women during their menstrual years (and longer if estrogen replacement therapy is used).

Low levels of transferrin are commonly seen in inflammation or malignancy. Decreased synthesis is seen with chronic liver disease and malnutrition. Protein loss, as in the nephrotic syndrome or protein-losing enteropathies, also results in low levels. In iron deficiency, transferrin is increased, but the protein is less saturated with iron.

In iron overload (e.g., hereditary haemochromatosis), transferrin concentration is normal (30-38\%), but saturation may be as great as $100 \%$. Assays of soluble transferrin receptor are useful in differentiating the various causes of microcytic anaemia; if tissue iron deficiency is present, the affected cells increase their synthesis of transferrin receptor. High levels of transferrin are also seen in pregnancy and during estrogen administration.

\section{CONCLUSION}

This study established that the plasma levels of ceruloplasmin and transferrin were significantly increased and decreased respectively in Nigerian diabetes mellitus patients studied. Hence, ceruloplasmin and transferrin levels may be useful markers in monitoring and controlling diabetes mellitus disease.

\section{REFERENCES}

ADA (American Diabetes Association), 2000. Type II diabetes in children and adolescents. Diabetes Care, 23: 381-389.
Cheesbrough, M., 2000. Clinical chemistry test. District Laboratory Practical in Tropical countries. Edinburgh, pp: 341.

Fahey, J.L. and E.M. Mckelvey, 1965. Quantitative determination of serum immunoglobulins in antibody agar plate. J. Immunol., 94: 84-90.

Killingsworth, L.M., 1979. Plasma protein patterns in health and diseases. Crit. Rev. Clin. Lab. Sci., 11: 1-30.

Metzger, B.E. and D.R. Coustan, 1998. Summary and recommendations of the fourth international workshop. Conference on Gestational Diabetes Mellitus. Diabetes Care, 21: 161-167.

Ponder, S.W., S. Sullivan and G. McBath, 2000. Type II diabetes mellitus Teens. Diabetes Spectrum, 13: 95.

Ramaza, M.O. and B. Ebukekir, 2004. Levels of ceruplasmin, transferrin and lipid peroxidation in the serum of patients with Type II diabetes mellitus and its Complications. J. Diabetes, 10: 193-197.

Salimonu, L.S., O.A. Lapido, S.O. Adeniran and B.O. Osunkoya, 1978. Serum immunoglobulin levels in normal, premature postmature babies and their mothers. Int. J. Gyn. Obstet., 16: 119-123.

Van Eden, W. and D.B. Young, 1995. Stress protein in medicine. Marcel Dekker, 12: 132-141.

WHO (World Health Organisation), 1985. Diabetes mellitus: Report of a WHO study group. Geneva, pp: 27.

WHO (World Health Organization), 1999. Booklet Laboratory diagnosis and monitoring of diabetes mellitus, pp: 27.

Yoshida, K., K. Furihata and S. Takeda, 1995. A mutation in the ceruloplasmin gene is associated with systemic haemosiderosis in humans. Nat. Genet., 9: 267-272.

Yonekawa, M., T. Okabe and M. Ohta, 1999. A case of hereditary ceruloplasmin with iron deposition in the brain associated with chorea, dermentia, diabetes mellitus and retinal pigmentation: Administration of fresh-frozen human plasma. Euv. Neurol., 42: 157-162. 